\title{
Effects of Clearance between Rotor and Ground on the Performance of an Open Cross-Flow-Type Nano-Hydraulic Turbine
}

\author{
Tomomi Uchiyama ${ }^{1}$, Shouhei Mizoguchi ${ }^{2}$, Shouichiro Iio $^{3}$, Yusuke Katayama ${ }^{3}$ and Toshihiko Ikeda ${ }^{3}$ \\ 1. Institute for Materials and Systems for Sustainability, Nagoya University, Nagoya 464-8603, Japan \\ 2. Toyota Motor Corporation, Toyota 471-8571, Japan \\ 3. Faculty of Engineering, Shinshu University, Nagano 380-8553, Japan
}

Received: April 11, 2016 / Accepted: May 5, 2016 / Published: August 31, 2016.

\begin{abstract}
Flows through an open cross-flow-type nano-hydraulic turbine are numerically simulated to investigate the effects of the clearance $H_{C}$ between the rotor and the ground on the turbine performance. A two-dimensional particle method is employed, which was successfully used for flow simulations of impulse-type and open cross-flow-type nano-hydraulic turbines in the authors' previous works. When the clearance $H_{C}$ is smaller than a critical value, the simulated turbine performance decreases with decreasing $H_{C}$, in good agreement with the experiment. The simulations make it clear that such a reduction of turbine performance is attributable to an increase in the circumferential component of the water velocity at the rotor outlet. The simulations also demonstrate that the effect of the tip speed ratio of the rotor on the relation between $H_{C}$ and turbine performance can be analyzed.
\end{abstract}

Key words: Nano-hydraulic turbine, computer simulation, waterfall, particle method.

\section{Introduction}

In Japan, hydropower is a promising renewable energy resource, and there is increasing interest in the development of small-scale hydropower with output less than $100 \mathrm{~kW}$. This is because such hydropower, which is often located in small rivers, irrigation canals, and industrial drainage channels, is distributed widely and thus allows realization of distributed power generation.

Various hydraulic turbines for small-scale hydroelectric power generation have been proposed, and further developments to improve their performance have been reported [1-11]. The impulse-type [5] and open cross-flow-type [10, 11] hydraulic turbines use extra-low head waterfalls in small rivers and agricultural canals. These turbines are called nano-hydraulic

\footnotetext{
Corresponding author: Tomomi Uchiyama, professor, Dr. Eng., research fields: fluid engineering and CFD.
}

turbines, and their output is less than a few kilowatts. Such nano-hydraulic turbines are easily transported to the generation site and produce electricity without damaging the environment. According to laboratory experiments conducted by Katayama et al. [10] using their open cross-flow-type hydraulic turbine, the power output from the rotor is affected by the clearance between the rotor and the ground, and reducing the clearance below a certain threshold causes the output to decrease. Such performance deterioration is closely related to the water flow through the rotor, but the precise cause is not clear. To maximally utilize the head of a waterfall, placing the rotor closer to the ground is effective. Consequently, it is important to clarify the causes of the deterioration of the rotor output owing to the decrease in the clearance between the rotor and the ground.

Uchiyama et al. [12] performed a numerical simulation of the flow through the impulse-type 
nano-hydraulic turbine of Ikada et al. [5] and analyzed the turbine performance using the simulated flow field. For the simulation, the MPS (moving particle semi-implicit ) method [13, 14], which is one of the particle methods applicable to free-surface flows, was applied. The flow phenomena, such as the collision of the waterfall with the blades and the subsequent dispersion of water around the rotor, as well as the turbine performance, agreed well with the experimental results. Uchiyama et al. [15] also applied the MPS method to simulate the flow through an open cross-flow-type nano-hydraulic turbine driven by rapid and shallow stream. They confirmed that the simulated flow and turbine performance almost agree with the experimental results, demonstrating that the MPS method is also applicable to the design of such nano-hydraulic turbines. Moreover, Uchiyama et al. [16] simulated the flow through an open cross-flow-type nano-hydraulic turbine driven by a waterfall to clarify the causes for the decrease in turbine performance with increasing water flow rate.

In this study, the flows through an open cross-flow-type nano-hydraulic turbine driven by an extra-low head waterfall [10] are simulated by the MPS method, and the effects of the clearance between the rotor and the ground on the turbine performance are investigated. The decrease in the power coefficient with decreasing the clearance, which was observed experimentally by Katayama et al. [10], is successfully analyzed. The simulation also makes it clear that the decrease is caused by the increase in the circumferential component of the water velocity at the rotor outlet.

\section{Basic Equations}

\subsection{Conservation Equations for Flow}

If the flow through the hydraulic turbine is incompressible, it is governed by the mass and momentum conservation equations:

$$
\frac{\mathrm{D} \rho}{\mathrm{D} t}=0
$$

$$
\frac{\mathrm{D} \boldsymbol{u}}{\mathrm{D} t}=-\frac{1}{\rho} \nabla p+\nu \nabla^{2} \boldsymbol{u}+\boldsymbol{F}
$$

where, $\rho$ is the density, $t$ is the time, $\boldsymbol{u}$ is the velocity, $p$ is the pressure, $v$ is the kinematic viscosity, and $\boldsymbol{F}$ is the total external force including the gravitational force and surface tension.

This study analyzes Eqs. (1) and (2) by using the MPS method [13, 14]. In the MPS method, the fluid is discretized by the particles and the particle motion is computed by the Lagrangian method. Eqs. (1) and (2) are discretized through the interactions between the particles.

\subsection{Particle Interaction Model}

The interactions between the particles are modeled by using the weight function, $w$ which is defined by the following equation:

$$
w(r)=\left\{\begin{array}{cc}
r_{e} / r-1 & r \leq r_{e} \\
0 & r>r_{e}
\end{array}\right.
$$

where, $r$ is the distance between two particles and $r_{e}$ is the kernel size.

The particle number density at the position of the $i$ th particle, $\langle n\rangle_{i}$, is defined as

$$
\langle n\rangle_{i}=\sum_{j \neq i} w\left(\left|\boldsymbol{r}_{j}-\boldsymbol{r}_{i}\right|\right)
$$

where, $\boldsymbol{r}_{i}$ is the position vector of the $i$ th particle.

As the fluid density is constant for incompressible flow, the particle number density is also required to be constant. The incompressible flow condition in the MPS method is satisfied by maintaining $\langle n\rangle_{i}$ at a constant value $n^{0}$.

The Laplacian operator, expressing the viscous term on the right side of Eq. (2), is modeled with the weight function. The Laplacian operator at the position of the $i$ th particle is given as

$$
\left\langle\nabla^{2} \phi\right\rangle_{i}=\frac{2 d}{n^{0} \Lambda} \sum_{j \neq i}\left[\left(\phi_{j}-\phi_{i}\right) w\left(\boldsymbol{r}_{j}-\boldsymbol{r}_{i}\right)\right]
$$


where, $\phi$ is a physical quantity. The parameter $\Lambda$ is introduced such that the variance increase is equal to the analytical solution

$$
\Lambda=\int_{V} w(r) r^{2} \mathrm{~d} v / \int_{V} w(r) \mathrm{d} v
$$

The gradient operator, expressing the pressure gradient term on the right side of Eq. (2), is also modeled. The gradient operator at the position of the $i$ th particle is modeled by setting the interparticle force equal to the repulsion force to ensure numerical stability. Thus, the interacting pressure forces between the particles are not antisymmetric and the momentum is not always conserved. To solve this problem, the gradient operator model proposed by Khayyaer and Gotoh [17] is employed. The gradient operator is expressed as follows:

$$
\langle\nabla \phi\rangle_{i}=\frac{d}{n^{0}} \sum_{j \neq i}\left[\frac{\left(\phi_{i}+\phi_{j}\right)-\left(\hat{\phi}_{i}+\hat{\phi}_{j}\right)}{\left|\boldsymbol{r}_{j}-\boldsymbol{r}_{i}\right|^{2}}\left(\boldsymbol{r}_{j}-\boldsymbol{r}_{i}\right) w\left(\left|\boldsymbol{r}_{j}-\boldsymbol{r}_{i}\right|\right)\right]
$$

where, $d$ is the number of spatial dimensions and $\hat{\phi}_{i}$ is defined as

$$
\hat{\phi}_{i}=\min _{j \in J}\left(\phi_{i}, \phi_{j}\right), J=\left\{j: w\left(\left|\boldsymbol{r}_{j}-\boldsymbol{r}_{i}\right|\right) \neq 0\right\}
$$

\subsection{Simulation Method}

Eqs. (1) and (2) are solved by a semi-implicit method used within the SMAC (simplified marker and cell) method [18]. If the particle velocity $\boldsymbol{u}_{i}{ }^{k}$ and position $\boldsymbol{r}_{i}{ }^{k}$ at time $t=k \Delta t$ are known, the flow at time $t=(k+1) \Delta t$ is simulated by the following two steps.

In the first step, the temporal velocity and position of the particle, $\boldsymbol{u}_{i}{ }^{*}$ and $\boldsymbol{r}_{i}{ }^{*}$, respectively, are calculated from Eq. (2) without considering the pressure gradient term. Then, the temporal particle number density $\left\langle n^{*}\right\rangle_{i}$ is computed using Eq. (4).

In the second step, the following Poisson equation is solved for the pressure $p^{k+1}$, so that the mass conservation is satisfied or $\left\langle n^{*}\right\rangle_{i}$ is made to coincide with $n^{0}$.

$$
\left\langle\nabla^{2} p^{k+1}\right\rangle_{i}=-\frac{\rho}{\Delta t^{2}} \frac{\left\langle n^{*}\right\rangle_{i}-n^{0}}{n^{0}}
$$

Then, the temporal velocity and position of the particle are corrected by the obtained pressure gradient.

$$
\begin{gathered}
\boldsymbol{u}_{i}^{k+1}=\boldsymbol{u}_{i}^{*}+\boldsymbol{u}_{i}^{\prime} \\
\boldsymbol{r}_{i}^{k+1}=\boldsymbol{r}_{i}^{*}+\boldsymbol{u}_{i}^{\prime} \Delta t
\end{gathered}
$$

where,

$$
\boldsymbol{u}_{i}^{\prime}=-\frac{\Delta t}{\rho}\left\langle\nabla p^{k+1}\right\rangle_{i}
$$

The viscous term in Eq. (2) and the left side of Eq. (9) are computed using the Laplacian operator, Eq. (5). The right side of Eq. (12) is calculated using the gradient operator, Eq. (7).

\section{Simulation Conditions}

This study simulates the flows through an open cross-flow-type nano-hydraulic turbine driven by an extra-low head waterfall. The turbine performance was investigated by laboratory experiments [10, 11]. Fig. 1 shows the rotor and blade specifications, where the

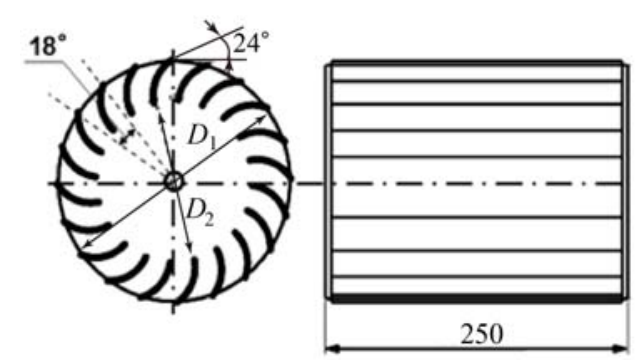

Rotor

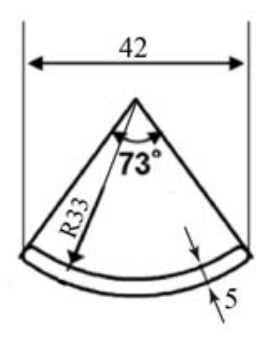

Blade

Fig. 1 Rotor and blade specifications. 
rotor outer diameter $D_{1}$ is $200 \mathrm{~mm}$, the rotor inner diameter $D_{2}$ is $132 \mathrm{~mm}$, and the number of blades is 20 . Each blade has a circular-arc shape, a curvature radius of $33 \mathrm{~mm}$, a thickness of $5 \mathrm{~mm}$, and a chord length of $42 \mathrm{~mm}$.

In the experiments, the rotor is placed at the bottom of a waterfall, as shown in Fig. 2. The origin of the coordinates is set at the rotor axis. The $\mathrm{X}$-axis is horizontal, and the Z-axis is vertical. The origin of the polar coordinates $(r, \theta)$ is also set at the rotor axis. According to the experiments, the turbine efficiency is maximized at a flow rate $Q$ of $0.003 \mathrm{~m}^{3} / \mathrm{s}$. In the present study, the flow at this flow rate is simulated using the two-dimensional MPS method to explore the effects of the clearance $H_{C}$ between the rotor and the ground on the turbine efficiency. The head of the waterfall $H_{T}$ is $710 \mathrm{~mm}$, and the distance $L_{C}$ between the waterfall and the rotor axis is $L_{C} / D_{1}=0.43$. The ground is horizontal, which is similar to the case in the experiments. The simulation conditions are summarized in Table 1.

A rectangular region around the rotor is chosen as the computational domain. The region is shown by a broken line in Fig. 2. The width in the horizontal $(x)$ direction is $2.5 D_{1}$, and the vertical height is $1.25 D_{1}+$ $H_{C}$. The waterfall flows into the computational domain from location A on the upper boundary. The particles,

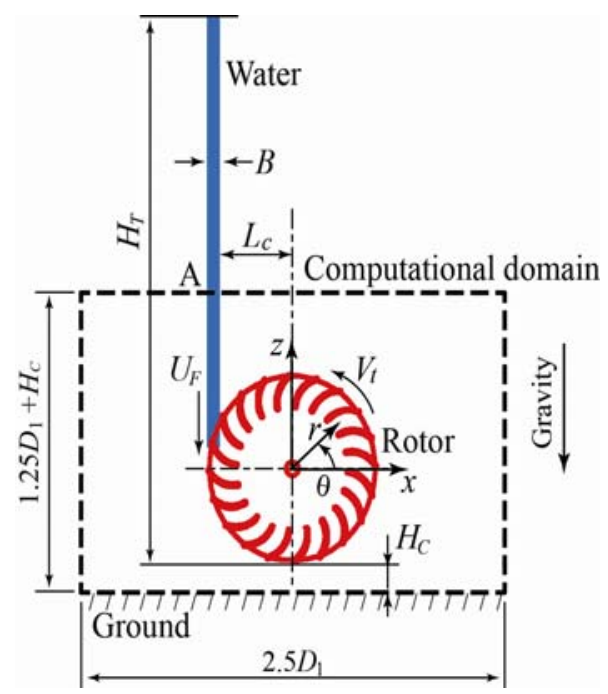

Fig. 2 Computational domain and positional relation between waterfall and rotor.
Table 1 Simulation conditions

\begin{tabular}{ll}
\hline Water flow rate $Q$ & $0.003 \mathrm{~m}^{3} / \mathrm{s}$ \\
Head of waterfall $H_{T}$ & $0.71 \mathrm{~m}$ \\
Thickness of waterfall $B$ & $0.004 \mathrm{~m}$ \\
Outer diameter of rotor $D_{1}$ & $0.2 \mathrm{~m}$ \\
Inner diameter of rotor $D_{2}$ & $0.132 \mathrm{~m}$ \\
Number of blades & 20 \\
Clearance between rotor and ground $H_{C} / D_{1}$ & $0.015-0.15$ \\
\hline
\end{tabular}

which discretize the falling water, are released from location A into the domain at a velocity determined from free-falling conditions. In order to ensure computational accuracy, the released particles must be arranged uniformly in the horizontal and vertical directions. At location $\mathrm{A}$, the particles are arranged at an interval $l_{0}$ in the horizontal direction so that the width of waterfall $B$ coincides with the measured value $(4 \mathrm{~mm})$. The particles are released into the domain at a constant time interval $\Delta t_{p}$, which is set such that the vertical distance between the particles is $l_{0}$.

The rotor axis and blades form the solid walls, which are discretized by particles having the same angular velocity as the rotor. The distance between these particles is also set at $l_{0}$. The Neumann boundary condition for the pressure gradient is imposed on the particles contacting with the fluid.

The pressure is set to zero on the free surface, the position of which is determined according to the value of the particle number density. When the particle number density, $\left\langle n^{*}\right\rangle_{i}$, obtained from the first calculation step in each time step, satisfies the following relation, the $i$ th particle is deemed to be on the free surface

$$
\left\langle n^{*}\right\rangle_{i}<\beta n^{0}
$$

where, $\beta$ is a parameter and $\beta<1$.

The time increment $\Delta t$ is set at $5 \times 10^{-5} \mathrm{~s}$. The value of $r_{e}$ in Eq. (3) is generally chosen such that $2 \leq r_{e} / l_{0}$ $\leq 4[13,14]$. The value of $r_{e}$ is set at $2.1 l_{0}$ for the particle number density and gradient operator, and at 4 $I_{0}$ for the Laplacian operator [14]. The parameter $\beta$ in Eq. (13) is set at 0.97 . 


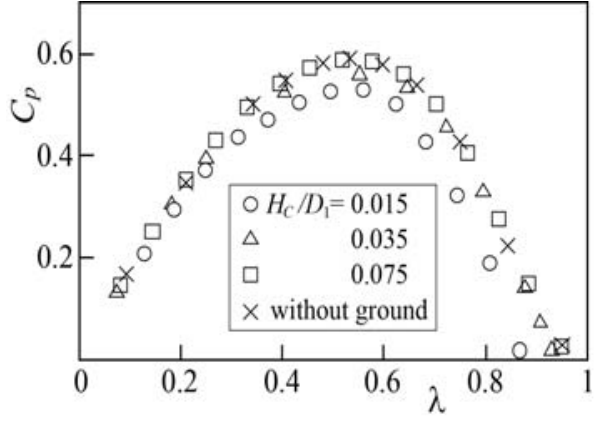

Fig. 3 Experimental results for the dependence of power coefficient on clearance between rotor and ground.

The tip speed ratio $\lambda\left(=V_{t} / U_{F}\right)$ is defined as the ratio of the rotor tip speed $V_{t}\left(=\omega D_{1} / 2\right)$ to the impact velocity of the waterfall with the blade $U_{F}$, where $\omega$ is the angular velocity of the rotor.

The power output from the rotor is expressed as the dimensionless power coefficient $C_{p}$.

$$
C_{p}=\frac{\left(T_{1}-T_{2}\right) \omega}{\frac{1}{2} \rho Q U_{F}^{2}}
$$

where, $T_{1}$ and $T_{2}$ are the torques on the outer and inner peripheries of the rotor, respectively, and are calculated by the following equation.

$$
T_{i}=\int \rho\left(\frac{D_{i}}{2}\right)^{2} u_{r i} u_{\theta i} \mathrm{~d} \theta, \quad(i=1,2)
$$

where the subscripts 1 and 2 denote the outer and inner peripheries, respectively. Here $u_{r}$ and $u_{\theta}$ are the radial and circumferential components of the water velocity, respectively. The sign of $u_{r}$ is defined to be positive for the radially inward flow and that of $u_{\theta}$ is also defined to be positive for the stream flowing in the same rotational direction as the rotor.

The relation between $\lambda$ and $C_{p}$ obtained experimentally by Katayama et al. [10] is shown in Fig. 3 . When the clearance $H_{C}$ between the rotor and ground satisfies the relation $H_{C} / D_{1}<0.075, C_{p}$ decreases with decreasing $H_{C} . C_{p}$ takes the maximum value at $\lambda \cong 0.55$ irrespective of $H_{C}$. First, this study demonstrates that the decrease in $C_{p}$ with decreasing $H_{C}$ can be simulated. Second, it clarifies the causes for the decrease in $C_{p}$ on the basis of the simulated flow field.

\section{Results and Discussions}

\subsection{Effect of $H_{C}$ on Turbine Performance and Flow Field}

The spatial resolution of this simulation is chiefly governed by the interval $l_{0}$ between the particles discretizing the flow. First, the effect of $l_{0}$ on the simulation results is explored.

The flow through the rotor is simulated to calculate the torque $T$ from Eq. (15), and then the power coefficient $C_{p}$ is estimated from Eq. (14). Fig. 4 shows the dependence of $C_{p}$ on the clearance $H_{C}$ between the rotor and the ground, where the tip speed ratio $\lambda$ is 0.55 . In the case of $l_{0} / B=0.25, C_{p}$ decreases with decreasing $H_{C} / D_{1}$ when $H_{C} / D_{1}<0.075$. Though this change agrees with the experimental results indicated in Fig. 4, the $C_{p}$ value is larger than in the experiments. When $l_{0}$ is decreased to $l_{0} / B=0.125, C_{p}$ reduces and approaches that from the experiments, almost agreeing with the experiments at $H_{C} / D_{1}=0.015$ and 0.035 . This is because the number of particles increases for smaller $l_{0}$, and accordingly, the spatial resolution is improved. The simulation of much smaller $l_{0}\left(l_{0} / B=0.1\right)$ is also performed at $H_{C} / D_{1}=0.075$. The $C_{p}$ value is almost the same as that for $l_{0} / B=0.125$, as plotted with the symbol "+” in Fig. 4. This suggests that the simulation of $l_{0} / B=0.125$ ensures a sufficiently high spatial resolution. Thus, this study discusses the results simulated with $l_{0} / B=0.125$.

Fig. 5 shows the particle distribution for the fully developed flow, where $\lambda=0.55$. Fig. 5 a shows the distribution when the clearance $H_{C}$ is at the maximum $\left(H_{C} / D_{1}=0.15\right) . C_{p}$ is not affected by the clearance, as found in Fig. 3. The waterfall collides directly with the concave surface of the blade. After collision, the water flows toward the inside and outside of the rotor as the rotor rotates. The water flowing toward the outside disperses, and the water directed toward the inside flows along the concave surface of the blade and then enters the inside of the rotor. The flow rate toward the inside is much larger. The water inside the rotor collides again with the concave surface of the blade 


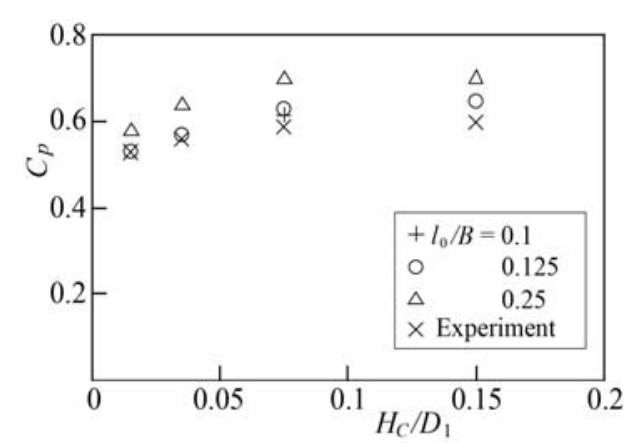

Fig. 4 Dependence of power coefficient on clearance between rotor and ground at $\lambda=\mathbf{0 . 5 5 3}$.

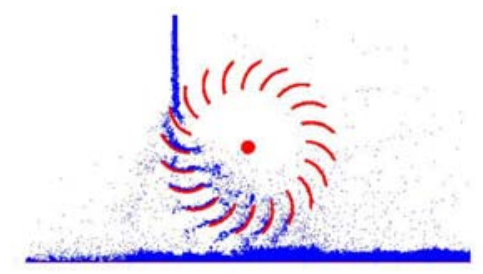

(a) $H_{C} / D_{1}=0.15$

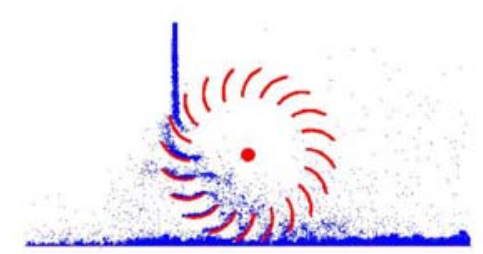

(b) $H_{C} / D_{1}=0.015$

Fig. 5 Particle distribution for fully developed flow at $\lambda=$ 0.55 .

and flows out of the rotor. The water flowing out of the rotor freely falls toward the ground. Consequently, this simulation highlights the following three kinds of flow: the waterfall flowing along the concave surface of the blade (1st-stage flow), the flow crossing inside the rotor (cross-flow), and the cross-flow flowing along the concave surface of the blade (2nd-stage flow). Fig. $5 \mathrm{~b}$ shows the particle distribution when the clearance $H_{C}$ is at the minimum $\left(H_{C} / D_{1}=0.015\right) . C_{p}$ is reduced due to the decrease in $H_{C}$, as found in Fig. 3. The 1st-stage flow, the cross-flow, and the 2nd-stage flow are almost the same as those at $H_{C} / D_{1}=0.15$ shown in Fig. 5a; however, the water flowing out of the rotor immediately joins the horizontal flow along the ground. The tip of the rotating blade drives the water in the right direction along the ground.
For the simulation of one revolution of the rotor at $H_{C} / D_{1}=0.015,18.6$ hours are required on a workstation (Processor: Intel Xeon X5660 2.8GHz $\times$ 6; Memory: $12 \mathrm{~GB})$. The number of particles is about 31,000 .

\subsection{Time-Averaged Velocity}

Fig. 6 shows the time-averaged water velocity distribution for $\lambda=0.55$. The distribution at $H_{C} / D_{1}=$ 0.15 is shown in Fig. 6a. At the outer periphery of the rotor, the waterfall either disperses toward the outside of the rotor or flows radially inward between the blades. Most water passes between the blades and flows into the rotor, producing the cross-flow inside the inner periphery of the rotor. The cross-flow then passes radially outward between the blades. Fig. $6 \mathrm{~b}$ shows the result at $H_{C} / D_{1}=0.015$. The 1 st-stage flow, the cross-flow, and the 2nd-stage flow are observed. When compared with the results at $H_{C} / D_{1}=0.15$ shown in Fig. $6 \mathrm{a}$, the velocity of the 2nd-stage flow at the rotor outlet is higher. This is because the rotating blade drives the

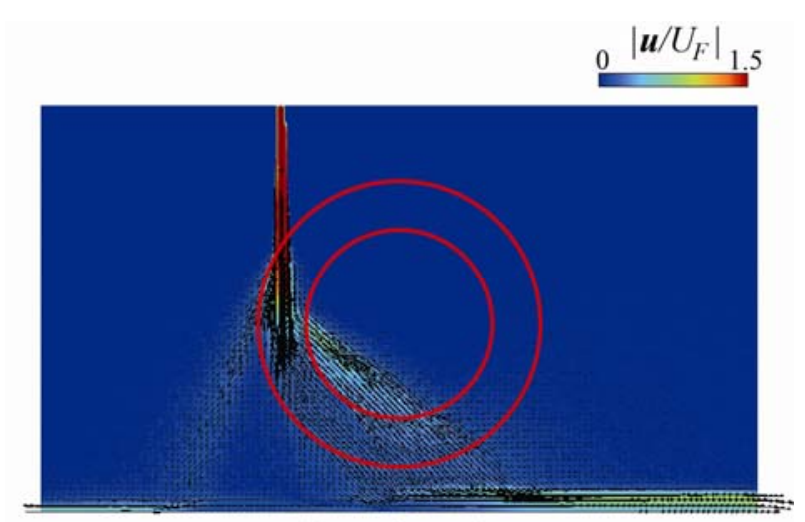

(a) $H_{C} / D_{1}=0.15$

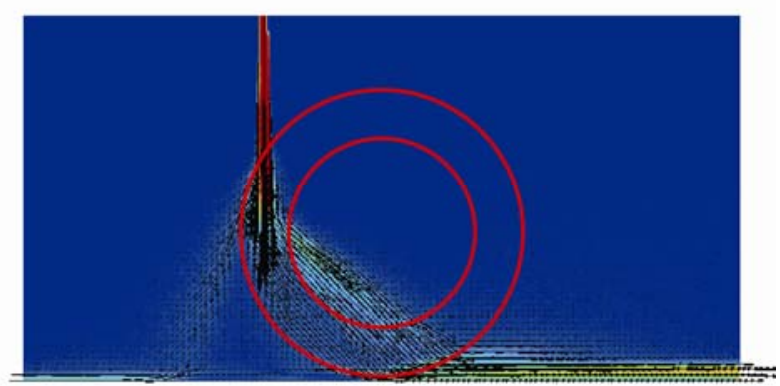

(b) $H_{C} / D_{1}=0.015$

Fig. 6 Time-averaged velocity distribution at $\lambda=0.55$. 


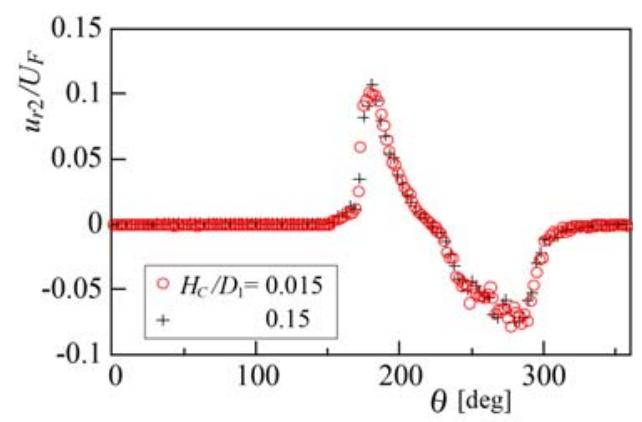

(a)

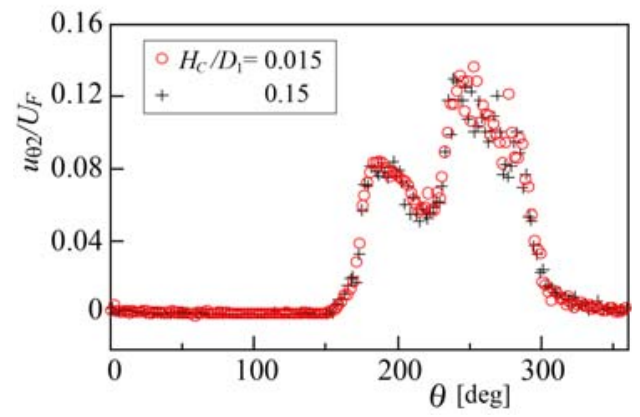

(b)

Fig. 7 Distribution of water velocity along inner periphery of rotor.

water on the ground in the right direction, increasing the velocity of the 2nd-stage flow at the rotor outlet.

Fig. 7 shows the distributions of the time-averaged velocity along the inner periphery of the rotor for $H_{C} / D_{1}=0.015$ and 0.15 . The radial velocity component $u_{r 2}$ is positive at $152 \leq \theta \leq 221 \mathrm{deg}$ and reaches a maximum value at $\theta=180 \mathrm{deg}$, where the inward flow between the blades enters the inner region of the rotor. This corresponds to the beginning of the cross-flow in the inner region of the rotor. At $222 \leq$ $\theta \leq 350$ deg, $u_{r 2}$ is negative. This indicates that the cross-flow in the rotor enters the region between the blades as the 2nd-stage flow. Hardly any effect of $H_{C}$ on $u_{r 2}$ is found. The circumferential velocity component $u_{\theta 2}$ reaches positive maximum values at the positions where the 1st-stage and 2nd-stage flows appear. The distribution of $u_{\theta 2}$ is also barely affected by $H_{C}$.

Fig. 8 shows the distributions of the time-averaged velocity along the outer periphery of the rotor for $H_{C} / D_{1}=0.015$ and 0.15 . The radial velocity component $u_{r 1}$ reaches a positive maximum value at $\theta=145 \mathrm{deg}$, where the waterfall collides with the blade, but is

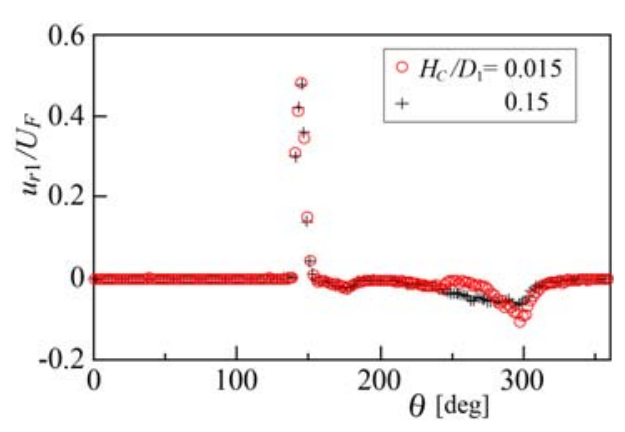

(a)

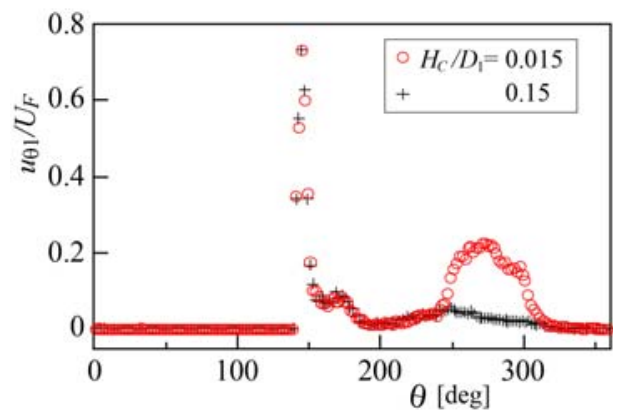

(b)

Fig. 8 Distribution of water velocity along outer periphery of rotor.

negative at $\theta \geq 155 \mathrm{deg}$. This corresponds with the fact that the water flows out of the rotor at $\theta \geq 155 \mathrm{deg}$. The effect of $H_{C}$ on $u_{r 1}$ is small. The circumferential component $u_{\theta 1}$ also reaches a positive maximum value at $\theta=145 \mathrm{deg}$, but is positive at $\theta \geq 132 \mathrm{deg}$ in this case. The effect of $H_{C}$ is marked for $242 \leq \theta \leq 312$ deg. In the case of $H_{C} / D_{1}=0.015, u_{\theta 1}$ is rather large. For $242 \leq \theta \leq 312 \mathrm{deg}$, the water flowing out of the rotor joins the water flow on the ground, as found in Figs. 5a and 6a. The outer periphery of the rotor is close to the ground, as observed in Fig. 6a, and the rotating blades accelerate the water along the ground, which affects the outlet velocity of the water at the rotor periphery. These result in the velocity component $u_{\theta 1}$ being higher at $H_{C} / D_{1}=0.015$.

\subsection{Torque at the Inner and Outer Peripheries of the} Rotor

The torques at the inner and outer peripheries of the rotor are calculated from Eq. (15) using the time-averaged water velocity shown in Figs. 7 and 8. When the peripheries are circumferentially divided into 
180 domains to calculate the torque $\Delta T$ at each domain, the nondimensional values $\Delta T^{*}\left(=\Delta T \omega /\left(\rho Q U_{F}^{2} / 2\right)\right.$ distribute as shown in Fig. 9, in which the distributions at $H_{C} / D_{1}=0.015$ and 0.15 are compared. Fig. 9a shows the distribution along the inner periphery. The torques produced by the 1st-stage and 2nd-stage flows are confirmed and are less affected by $H_{C}$. This is because $H_{C}$ hardly affects the water velocity distribution, as demonstrated in Fig. 7. Fig. 9b shows the torque distribution along the outer periphery. The torque reaches a higher value at $\theta=145 \mathrm{deg}$, where the waterfall collides with the blade. The maximum value does not depend on $H_{C}$. In the case of $H_{C} / D_{1}=0.15$, the torque is almost zero, except for the position where the maximum value appears. This indicates that the 2nd-stage flow at the outer periphery of the rotor does not give the energy to the rotor. For $262 \leq \theta \leq 312$ deg, however, the torque takes a negative value, and the absolute value is large in the case of $H_{C} / D_{1}=0.015$. Fig. 10 shows an enlarged distribution of this region. One can confirm that the torque reaches a minimum

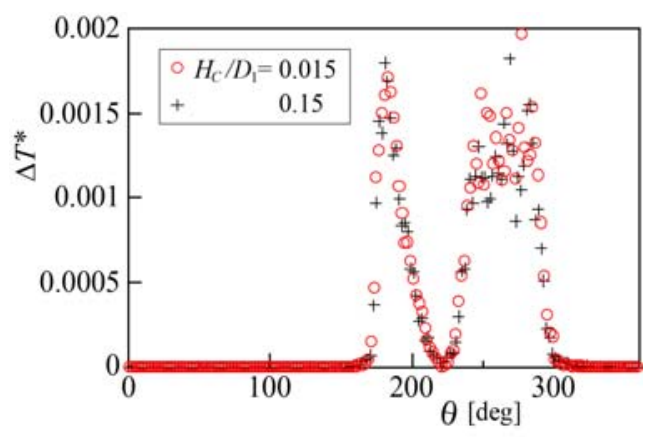

(a) Distribution along inner periphery

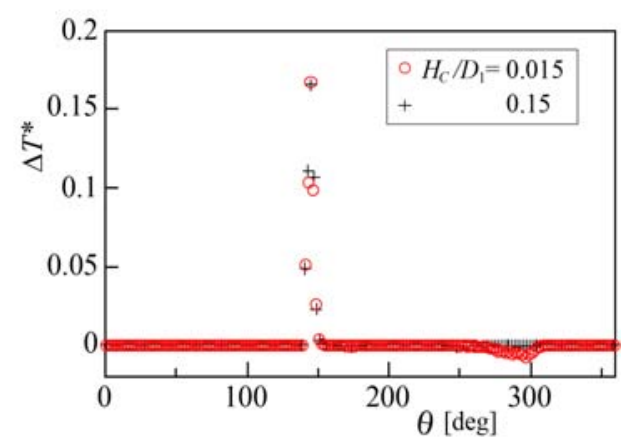

(b) Distribution along outer periphery

Fig. 9 Distribution of torque acting on inner and outer peripheries of rotor.

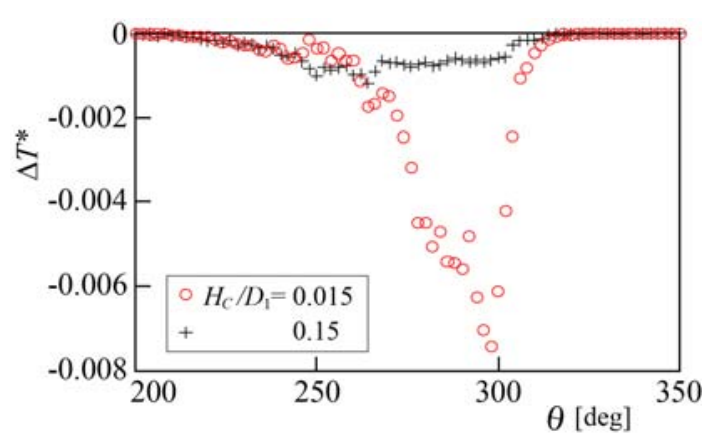

Fig. 10 Distribution of torque acting on outer periphery of rotor for $200 \leq \theta \leq 350 \mathrm{deg}$.

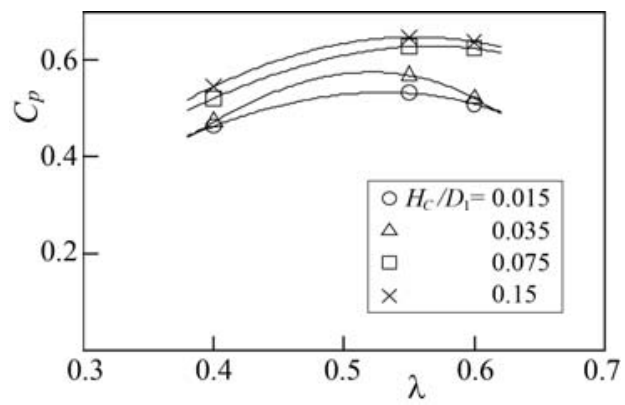

Fig. 11 Power coefficients simulated at $\lambda=0.4,0.55$, and 0.6.

negative value at $\theta=297 \mathrm{deg}$. Owing to such torque distributions, $C_{p}$ is greatly reduced in the case of $H_{C} / D_{1}$ $=0.015$, as shown in Fig. 4 . The torque is the product of the radial and circumferential velocity components of the water velocity, $u_{r 1}$ and $u_{\theta 1}$, respectively, as found from Eq. (15). The reason the torque is negative at $H_{C} / D_{1}=0.015$ is that $u_{r 1}$ and $u_{\theta 1}$ take negative and positive values, respectively, as shown in Fig. 8. The rotating blades accelerate the water on the ground along the ground, and therefore, the accelerated flow produces such velocity distribution at the outer periphery of the rotor.

4.4 Turbine Performance at Tip Speed Ratios $\lambda=0.4$ and 0.6

The simulation is also performed at tip speed ratios of $\lambda=0.4$ and 0.6. Fig. 11 shows the power coefficient $C_{p} . C_{p}$ decreases with decreasing the clearance $H_{C}$ between the rotor and the ground. Such a change due to $H_{C}$ coincides with the experimental results shown in Fig. 3. This demonstrates that the present simulation successfully analyzes the effect of $\lambda$ on the relation between $H_{C}$ and $C_{p}$. 


\section{Conclusions}

The flows through an open cross-flow-type nano-hydraulic turbine driven by an extra-low head waterfall were simulated by a two-dimensional particle method. The turbine performance was also analyzed based on the simulated flow field. The results can be summarized as follows:

When the clearance $H_{C}$ between the rotor and the ground is smaller than a threshold, the power coefficient $C_{p}$ decreases with decreasing $H_{C}$. This change coincides with the experimental results.

The simulation reveals that the decrease in $C_{p}$ with decreasing $H_{C}$ is caused by the water velocity at the outer periphery of the rotor. When $H_{C}$ is small, the rotating blades accelerate the water on the ground and increase the circumferential component of the water velocity at the outer tip of the blade, reducing the torque at the outer periphery of the rotor.

The present simulation successfully analyzes the effect of the tip speed ratio $\lambda$ on the relation between $H_{C}$ and $C_{p}$.

\section{References}

[1] Singh, P., and Nestmann, F. 2000. "Experimental Optimization of a Free Vortex Propeller Runner for Microhydro Application.” Experimental Thermal and Fluid Science 33 (6): 991-1002.

[2] Alexander, K. V., Giddens, E. P., and Fuller, A. M. 2009. "Axial-Flow Turbines for Low Head Microhydro Systems.” Renewable Energy 34 (1): 35-47.

[3] Hayashi, N., Tanaka, A., Iio, S., Sato, E., and Ikeda, T. 2010. "Development of Open Type Cross-Flow Turbine Utilizing Rapid and Shallow Stream (Investigation of Blade Angle and Installation Condition).” Presented at the International Conference of Renewable Energy, Yokohama, Japan.

[4] Shimokawa, K., Furukawa, A., Okuma, K., Matsushita, D., and Watanabe, S. 2010. "Side-Wall Effect of Runner Casing on Performance of Darrieus-Type Hydro Turbine with Inlet Nozzle for Extra-Low Head Utilization." Science China Technological Sciences 53(1): 93-9.

[5] Ikeda, T., Iio, S., and Tatsuno, K. 2010. "Performance of Nano-Hydraulic Turbine Utilizing Waterfalls.” Renewable Energy 35 (1): 293-300.

[6] Singh, P., and Nestmann, F. 2011. "Experimental
Investigation of the Influence of Blade Height and Blade Number on the Performance of Low Head Axial Flow Turbines.” Renewable Energy 36 (1): 272-81.

[7] Stark, B. H., Ando, E., and Hartley, G. 2011. "Modelling and Performance of a Small Siphonic Hydropower System.” Renewable Energy 36 (9): 2451-64.

[8] Iio, S., Katayama, Y., Uchiyama, F., Sato, E., and Ikeda, T. 2011. "Influence of Setting Condition on Characteristics of Savonius Hydraulic Turbine with a Shield Plate.” Journal of Thermal Science 20 (3): 224-8.

[9] Nishi, Y., Inagaki, T., Li, Y., Omiya, R., and Fukutomi, J. 2014. "Study on an Undershot Cross-Flow Water Turbine.” Journal of Thermal Science 23 (3): 239-45.

[10] Katayama, Y., Iio, S., and Veerapun, S. 2014. "Effect of Runner Position on Performance for Open Type Cross-Flow Turbine Utilizing Waterfalls.” International Review of Mechanical Engineering 8 (6): 1012-6.

[11] Katayama, Y., Iio, S., Veerapun, S. and Uchiyama, T. 2015. "Investigation of Blade Angle of an Open Cross-Flow Runner.” International Journal of Turbo and Jet Engines 32 (1): 65-72.

[12] Uchiyama, T., Fukuhara, H., Iio, S., and Ikeda, T. 2013. "Numerical Simulation of Water Flow through a Nano-Hydraulic Turbine of Waterfall-Type by Particle Method.” International Journal of Rotating Machinery Article ID 473842.

[13] Koshizuka, S., and Oka, Y. 1996. "Moving Particle Semi-Implicit Method for Fragmentation of Incompressible Fluid.” Nuclear Science and Engineering 123 (3): 421-34.

[14] Koshizuka, S., Nobe, A., and Oka, Y. 1998. "Numerical Analysis of Breaking Waves Using the Moving Particle Semi-Implicit Method.” International Journal for Numerical Methods in Fluids 26 (April): 751-69.

[15] Uchiyama, T., Uehara, S., Iio, S., Ikeda, T., and Ide, Y. 2014. "Numerical Simulation of Water Flow through Nano-Hydraulic Turbine Driven by Rapid and Shallow Stream.” Journal of Energy and Power Engineering 8 (10): 1663-72.

[16] Uchiyama, T., Uehara, S., Fukuhara, H., Iio, S., and Ikeda, T. 2015. "Numerical Study on the Flow and Performance of an Open Cross-Flow Mini-Hydraulic Turbine.” In Proceedings of the Institution of Mechanical Engineers, Part A: Journal of Power and Energy, 968-77.

[17] Khayyer, A., and Gotoh, H. 2008. "Development of CMPS Method for Accurate Water-Surface Tracking in Breaking Waves.” Coastal Engineering Journal 50 (2): 179-207.

[18] Amsden, A. A., and Harlow, F. H. 1970. The SMAC Method: A Numerical Technique for Calculating Incompressible Fluid Flows. Los Alamos Scientific Laboratory Report LA-4370. 